\title{
Formalizing a multi-dimensional land management system: the stakeholders' perspective
}

\author{
Ruba Jaljolie ${ }^{1}$ and Sagi Dalyot ${ }^{1 *}$ \\ ${ }^{1}$ Mapping and Geo-Information Engineering, The Technion, Haifa 3200003, Israel; e-mail: sruba93@campus.technion.ac.il; \\ dalyot@technion.ac.il
}

KEY WORDS: 5D Modelling, 3D City Modelling, Land Management System, Cadastre, Sustainability, Delphi

\begin{abstract}
:
It is expected that by the year $2050,66 \%$ of the world's population will live in urban areas, necessitating the efficient management of land and urban space, namely the requirement for multi-dimensional land management systems (MLMS). In most countries, current LMS are two dimensional, representing the reality on a plane. This research aims at outlying guidelines designed for augmenting existing 2D LMS to multi-dimensional ones, by investigating theoretical (conceptual) and technical inferences related to the adding of the height dimension, the time dimension and the scale dimension (scale dimension may also refer to levels of details). Sustainable multi-purpose land management systems for serving various end-users is essential, therefore, our study is based on a Delphi questionnaire, which aims at understanding the perspective of diverse stakeholders and experts who may use LMS, as well as mapping their requirements and expectations from the LMS. Questions were categorized into two groups: i) theoretical, focusing on semantic and mathematical definitions; and, ii) technical, focusing on functionalities, databases, data collection, etc. As a preliminary assessment, responses of eight experts, from different fields and countries, to the questionnaire were analysed and summarized. The results are presented in this paper, including the main issues that experts pointed out, as well as suggested classes, fields, procedures and functionalities that might be required from a multi-purpose MLMS.
\end{abstract}

\section{INTRODUCTION}

Multi-dimensional land management system (MLMS) is a framework for achieving, handling and analysing the restrictions, responsibilities and rights (RRR) of land properties in space (Jaljolie et al., 2018), time and different levels of detail. As the population is rapidly growing, land resources become scarcer and more valuable. This leads to complex and dense urban construction, especially in industrial and commercial centers, which leads to overlap and integration of structures in space. Thus, enforcing the requirement for efficient usage of land, meaning that perspectives for exploiting land resources vertically, among others, need to be constructed. To achieve this, advanced LMS are necessary, such as offering techniques for modelling multi-dimensional data, enabling the spatial analysis of urban space and operating engineering applications. However, the time dimension, as well as having different levels of details (LODs), are integral parts of urban development, and thus the 3D LMS might operate better if it includes temporal and LODs aspects. The temporal aspect is important since new trends in land and real estate management continue to emerge (e.g., 18-hours city), in conjunction with continues changes in land cover (e.g., complex structures, infrastructure and streets), which necessitate persisting track and adaptations in land registration systems for handling time - alongside space. Moreover, discussions about sustainable multi-purpose MLMS for serving various end-users have been raised, which might require different LODs and granularities. These urgent demands from LMS coincide with advances in technology that enable high performance of comprehensive and rich spatial analysis, functionalities, queries, and process on large data volumes.

Current research on MLMS put most emphasis on various technical aspects, mainly in terms of database and visualization, such as modelling different time stamps of 3D spatial locations, creating change history maps and constructing data models in several LODs. Still, mapping the functionalities and prerequisites of MLMS is far from complete, where the clear objectives of these systems (i.e., services they should provide) are still required, mainly since they affect the systems' configuration and its data structure, together with the embedded functionalities and processes. Critical questions and change of ideas that will serve as working grounds are still not fully made and determined, such as 'Is the establishment of a real estate management system (serving for, e.g., taxation, transfer of ownership) is the final goal, or is it a tool that will serve other domains as well?". Also, will such a system be designed to support decision-making and applications needed for risk management, shadow estimation and utility networks administration? If yes, then which LODs are needed for each specific field involved in the MLMS, and which data update regularities are required? In addition to these issues, it is important to formalize uniform semantic and mathematical definitions of relevant terminologies that are based on the perceptions of the involved parties, i.e., the stakeholders, which also should be well defined. For example, the term "time dimension" in the land management context is not yet finalized, and it is not agreed whether a single dimension will be sufficient for handling complex aspects of time (Oosterom, Stoter, 2012), like: updates, analysis, scheduling and display (Langran, 1992); such that various different definitions are required to be illustrated.

Since MLMS is oriented for serving stakeholders and experts (among others), it is vital to understand their perspective regarding the above-described issues, and to qualitatively asses their expectations from a functional MLMS. It is also important to realize and model emerging unique and influential trends in real estate that directly affect land resources. For that, we designed a Delphi study, meaning an iterative questionnaire addressed to experts, for investigating and hence understating the future trends and data requirements that these experts expect from MLMSs. The experts participating in this study include the fields of real estate, urban planning, transportation, cadaster and geodesy. The questions are written in a manner that does not guide the respondent to a specific answer, but rather in a way that enables inferring as much information as possible. The questions are categorized under two groups: 1) Semantic and mathematical definitions focusing on the contributions, importance, and practicalities of MLMSs. For example, the question "How many dimensions are needed for describing temporal changes" is required since several studies consider 
temporal as more than a single dimension, as it may represent several sorts of changes, such as geometrical, topological, thematic or other changes related to the features of properties; 2) Data management, e.g., data structure and data model, functionalities and processes, data collection, accuracy, and visualization. A sample question in this context is: "Could you provide examples of continuous property changes that are important to track and supply for serving your discipline? What is the required tracking frequency?". The purpose of this question is to detect the required frequency of collecting and updating data in MLMSs. As an example, the answers received here vary: some disciplines demand only discrete information, e.g. tax authorities, while others need continuous change information, e.g. environmental monitoring organizations (as suggested in Krigsholm, et al., 2018).

This paper presents the preliminary outcome of this study, and the general guidelines that should be incorporated in the formalizing of MLMSs. Applications of LMS vary, for example, urban planning requires historical archiving, cultural heritage analysis and tracking urban development trends. On the other hand, applications for risk management in a natural disaster, need indoor 3D models in numerous LODs. These show that integrating temporal and scale aspects in MLSM need to be analyzed and well formalized beforehand. In our study, we aspire to firstly specify the disciplines that will use LMSs, and the way they will use it. According to these, we will recommend a formalization of all aspects required in an applicative MLMS, which will serve various experts from various fields having different needs. These will include, among others, data structure, processes and functionalities that are related to all dimensions. The technical framework of MLMS needs to be determined in advance, but it is also crucial to specify its longterm purposes and the appliances it offers.

\section{LITERATURE REVIEW}

\subsection{Time}

The topic of optimally storing and systematically managing continues changes in LMS is still undetermined. Different studies suggest different approaches, for example, (Nebiker et al., 2014) suggests using dense image matching and objectbased image analysis for creating change detections based on greyscale and colour aerial photographs, for the purpose of observing changes in landscape.

Multi-purpose LMS involves moving objects (or continuously evolving objects). For serving real estate purposes, the value of a property might be grasped as a moving financial object since it changes as a function of time, and it is not a geometric entity. For providing urban planning functionality, urban density can be understood as a semantic moving object, landowner is a moving object as well, since ownership may be transferred by inheritance or transactions. Earth surface and orthometric height also changes as a result of nature or human factors. (Güting, Schneider, 2005) provide examples of moving region entities, such as: countries that move as result of reunification, splitting into two (or more) parts and occupation; shrinking forests and lakes as a result of human or natural phenomenon; continental shifts. These examples show the need for treating moving objects in LMS by integrating the time dimension.

For managing the time dimension, using real-time databases is considered, which are defined as database systems that include all features on traditional database system; in addition to enforcing time-constraints in a form of data validation duration or transaction deadlines or both (Salem et al., 2018).

\subsection{LODs and scaling}

(Allard, 2009) suggests three approaches for presenting various scales in public transportation maps: 1 . Separate maps: users get separate maps in different scales (small- and large-scale maps) and can pick a map in the scale that fits their specific needs, but cannot see both maps concurrently. 2. Parallel maps (Insets): users get one primary map in a small scale, on which exists other inset larger-scale map that zoom in on a specific concentrated area 3. Variable scale maps: maps that present overcrowded areas in large-scales and less crowded areas in small-scales.

Various LODs (e.g., general cartographic / land cover model, models of buildings without texture, models of buildings in an area with texture, etc.) would be needed in LMS for performing typical processes and functionalities required in different fields. For example, in transportation field, navigators need general small-scale maps that depict whole scenes, as well as largerscale maps the details specific crowded areas (Allard, 2009), (Harrie et al., 2002).

Presenting data in various LODs involves technical and conceptual problems related to generalization. "It should be clear how former area objects are converted to line objects. For example, collapsing road polygons to lines might require additionally that former road areas are assigned to (which?) neighbouring area objects...Collapsing polygons to lines is not always trivial. For example, roundabouts are introduced at smaller scales as points because of the linear road network replacing road polygons. However, in $1: 10 \mathrm{~K}$ database roundabouts are not encoded, but can be interpreted by humans from the polygons" ( Stoter et al., 2011 744).

\section{METHODOLOGY}

As stated in the abstract, the purpose of this research is to outlay guidelines for augmenting existing 2D LMS to multidimensional ones, by investigating theoretical and technical inferences related to the adding of the height dimension, the time dimension and the scale dimension. For this goal, we pursue a Delphi approach, which is defined as an iterative survey, directed at specialists in the field, for understating the future trends affecting specific arena (in this case MLMSs), in which no working model exist. We adopted Delphi study since we seek to suggest an optimal structure of MLMS based on the perceptions of potential end-users. The diversity of the survey's participants is important for exposing different - and even contradicting - opinions regarding particular topic, as-well-as mapping common points, on which they all agree, hence, try to reveal their consensus - if exists - on matters involved in the survey. Starting from the common points, we provide suggestions related to the data structure (i.e., classes and fields), processes, functionalities and queries that need to be embedded into multi-purpose MLMS. We also stimulate new issues regarding the creation of MLMS that until now was not sufficiently emphasized. 


\subsection{Disciplines and stakeholders}

Two-parts Delphi questionnaire ${ }^{1}$ was published online among potential stakeholders of future 5D LMS. The feedbacks of 5 online participants were included in the analysis, as well as 3 personal interviews that were carried out. The 8 participants included - among others - experts of urban planning and real estate, Geographic Information Systems (GIS), spatial analysis and spatial science, municipalities engineer. Participants had generally long years of experience in academic and/or technical background, and were from various countries.

\section{2 potential end-users of MLM}

According to the responses, potential disciplines to use LMS include urban planning organizations, jurisdictional organizations (e.g. registration authorities), mapping and surveying institutions, municipalities, cadastre departments, real-estate market specialists, spatial analysts and GIS experts, transportation analysts and planners, real estate assessment and appraisal firms, etc.

\subsection{Main Consensuses}

Respondents had conflicting opinions regarding specific issues but agreed on others, like the need to combine agencies involved in mapping and registration processes, and the need to fit data structure to various end users and applications.

3.3.1 Combined agencies: Participants agreed that multipurpose systems that allow smooth collaboration - or even unification - between different organizations would be effective, sustainable and enable quicker procedures. Jurisdictional and mapping agencies are mostly separated, and combining these two institutions will provide more general and collective representation of the reality. Based on the experiences of the municipalities' engineering departments, it takes long time for an urban plan to be initiated, resulting in institutional delays (especially mapping and jurisdictional); had one uniform, smooth and online procedure existed for handling municipalities' applications, projects would advance faster and more effectively. However, even if uniform procedures existed, experts from at least two different fields are still required to be involved in such a process, for avoiding surplus complications. Both lawyers and land surveyors should take part, they must collaborate, but do not have to physically work in the same organization. To sum up, it is recommended to unify and combine processes and institutions, however, combination does not necessarily mean that the same person should be able to do all the tasks, neither it means working in the same physical space. Governments should take steps for combing these separated institutions, more research, or even a pilot may be required, for examining the efficiency of doing that.

\subsection{Other issues and examples}

The experts from different fields and backgrounds stated concerns that were not usually emphasized, such as the need of researching 3D urban planning instead of multi-dimensional land management or cadastral systems.

\footnotetext{
${ }^{1}$ Online survey: https://www.surveymonkey.com/r/88H85H5
}

3.4.1 3D registration vs. 3D urban planning: 3D registration systems are ineffective and cannot exist without 3D planning, which should be investigated, researched and implemented beforehand to investigating 3D registration. Even if the world have enabled 3D registration, it would not be helpful without 3D planning, since planning occurs before licensing and registrations.

\subsection{Time dimension}

Crucial or not? Based on the stakeholders perspectives, time dimension is crucial for specific applicants, but less important for others. The frequency of collecting data varies according to different fields. For example, effective urban planning requires real-time update of databases, while this is not required in cadastre. This is because there is almost no continuously evolving or continuously moving objects in cadastre. Though continuous update of the time dimension might not be always crucial for cadastre and registry, it is crucial for public transportation analysis and planning. Parameters, such as optimal public transportation frequency, bus stations' location and bus routes, depend on the surrounding: which buildings exist in a neighbourhood, how many departments are in each building, and how many people live in each department; these data are time dependent and utilise time-dimension.

The analysis of population growth is vital for future urban planning, such as determining the optimal location and density of public buildings in a neighbourhood. If databases hold dynamic information that represent construction density, population growth, urban trends, and other urban attributes of the last few years, an extrapolation can be used for predicting the same attributes for upcoming years. The question raised in this context is again about the required frequency of updating databases, and regarding the time resolution. The participants did not give a specific answer (value) to this issue, although they agreed that the time dimension is required in diverse resolutions for serving diverse users and application though.

3.5.1 Suggested approaches for updating databases: one approach suggests determining, beforehand, a rate of updating databases, which could be once a year, a month, a week - or any other rate - based on the different users' needs; data in between would be lost though.

Another approach recommends on flowingly and continuously updating databases each time a change is detected in the realworld. The second approach is much more comprehensive, and includes more data and details; however, data management might become more complicated. While this approach enables obtaining snapshots for any time stamp, the technical aspect and the volume of memory required for storing data should not be a concern. An example of practical utilization for this approach is reflected from engineering departments of municipalities, which suffer from the disability to track all the changes that take place in the land cover in their administration zones. Consequently, the citizens might not comply with the permitted construction rights - nor with the existing plans, and it is difficult for administrators to identify deviations; real-time update of LMS would help them to overcome this challenge.

\subsection{LODs dimension:}

Perspectives about the needed LODs varied. Most participants agreed that LODs are a function of applications and users. In urban areas, for example, the required LODs varies, where in 
dense areas more detailed LODs are required: a LOD that represent general whole areas, more detailed LOD representing neighbourhoods, much more detailed LOD representing specific features, such as buildings or roads. Tabular databases were suggested for holding entities in various LODs. Since visualization facilitates understanding of the reality, recommendations on linking tabular databases to GIS and visualization software were given.

\subsection{Technical aspects: procedures and data structure}

Based on the responses, classes, fields, functionalities and queries for handling 5D LMS were suggested.

\subsubsection{Classes:}

There is a need to create a "Transaction" class representing real transaction details. A "Transaction" should not be separated from an object (Figure 1), since a real transaction is an operation that regularly involves objects, and should be linked to them, which may represent a property (e.g., 3D parcel, apartment) or owners and ownerships of a property. The world may be represented by objects, while each object holds information about previous events it has undergone (statebased), or alternatively, by sequence of events that relate to different objects, while each event is an entity (event-based).

Respondents were asked whether they prefer to work within event-based or state-based model, and they expressed their desire to have both; from the one side, it is easier to understand observe the total picture of the reality based on objects, rather than based on transaction sequences. However, there are situations where registering "Transaction" is also crucial, and can exist by itself.

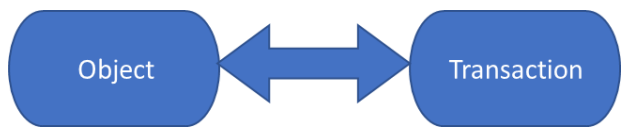

Figure 1. The "Transaction" class should be linked to objects.

An example for that was provided, involving an expropriation process. The law in Israel allows at most $40 \%$ cumulative land expropriation for public requirements without compensation; if previously $30 \%$ of a specific parcel area was expropriated, then a new plan can expropriate maximum additional $10 \%$ from the same original parcel. The Israel Land Authority and the Jerusalem municipality conflicted about the ratio allowed to be expropriated in a second expropriation for implementing a new urban plan. Had previous expropriation data was properly registered, the conflict could be avoided. For such circumstances, expropriation may be registered as a subclass of "Transaction". Depicted in Figure 2, the "Expropriation" class includes fields indicating the date of depositing the plan that impose expropriation, the purpose of expropriation, its percentage from the total parcel area, the cumulative expropriated area and the execution date (i.e. when expropriation was implemented).

\begin{tabular}{|l|}
\hline \multicolumn{1}{|c|}{ Expropriation } \\
\hline DepositingDate \\
ExecutionDate \\
ParcelsNumber \\
ExpropriationPurpose \\
ExpropriationPercentage \\
CummulativePercentage \\
\hline AddFieldsToExpropriation \\
GetFields \\
ReadFromCAD \\
\hline
\end{tabular}

\begin{tabular}{|l|}
\hline \multicolumn{1}{|c|}{ Transaction } \\
\hline TransactionDate \\
TransactionPurpose \\
\hline AddFieldsToExpropriation \\
GetFields \\
ReadFromCAD \\
\hline
\end{tabular}

Figure 2. An example for the "Expropriation" and "Transaction" classes.

\subsubsection{Fields:}

For proper time registration and track, respondents expressed that they would like to have both the valid time and the transaction time. The valid time refers to the time in the real world when an event occurs, or a fact that is valid. The transaction time refers to the time when a change is recorded in the database or the time interval during which a particular state of the database exists (Güting, Schneider, 2005). Therefore, regular classes that are composed of a data structure in $2 \mathrm{D}$ or 3D LMS should include two additional fields: "valid time" and "transaction time ". Valid time and transaction time enable stakeholders to understand gaps between registration, planning and reality. In addition, those fields make it easier to detect deviations from building permits. These two kinds of time can also serve for different purposes, transaction time may indicate when a change was registered, i.e., when the database was updated. A citizen who wants to purchase or sell real estate property needs an appraisal of the land. In some countries, such appraisal is based on previous transactions for similar properties located in the areas. Besides, the utilization of a property in the last years preceding the transaction date indicates the potentials of the property, which directly influence its value. For these reasons, chronological track of land changes is vital, end-users, especially appraisers, would like to receive up-to-date land information supplied with both transaction time and valid time. For managing LODs, regular traditional classes need to include fields that indicate the LOD of entities and instances of the class. Besides, an entity in a specific LOD should be linked to the same entity in other LODs, if exists. 


\subsubsection{Functionalities and process:}

Among others, two required processes for implementing urban plans were detected based on the responses, depicted in Figure 3 (Iterative interaction between registration and planning) and Figure 4 (Conventional stages prior to construction). Both processes integrate various end-users and show the need of multi-purpose LMS.

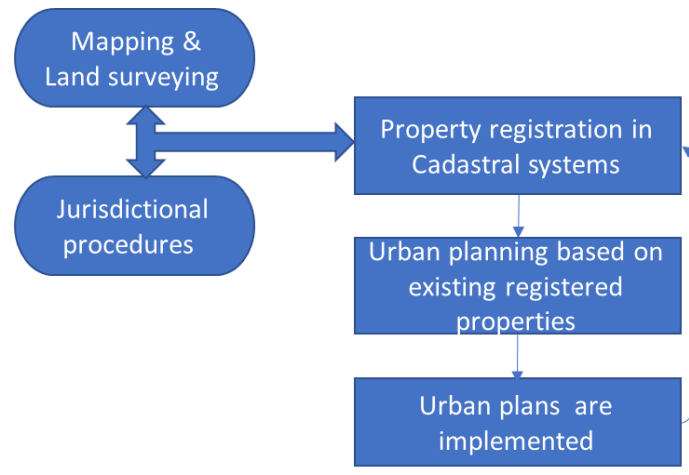

Figure 3. Iterative interaction between registration and planning.

Figure 3 illustrates an iterative process of registration and planning that involves different users and agencies. Prior to creating new urban plan, planners should consider the reality and base their plans on existing maps. Authorities hold maps of current state of their jurisdictional zone, ownerships of structures and parcels are regularly registered according to current state. If an urban plan is permitted, licensed and implemented, then it is required to register the changes that it caused. These mean that three iterative steps occur in the world of planning: (1) registering of existing properties; (2) new planning according to existing registration; (3) registering again. Implemented plans include vertical/horizontal split or union, construction of new apartments or building, with separate ownerships, land expropriations, and other operations that necessitate new registration.

In the process of registration, at least two types of agencies are involved: the mapping agency and the jurisdictional institution, where planning is performed by separate authorities (e.g., municipalities or governments). Changes that occur in one agency, as well as databases updating, affect the other agencies, which necessitate updating data in different agencies simultaneously. This encourage combining agencies work for performing proper, uniform and smooth procedures without delays and without conflict.

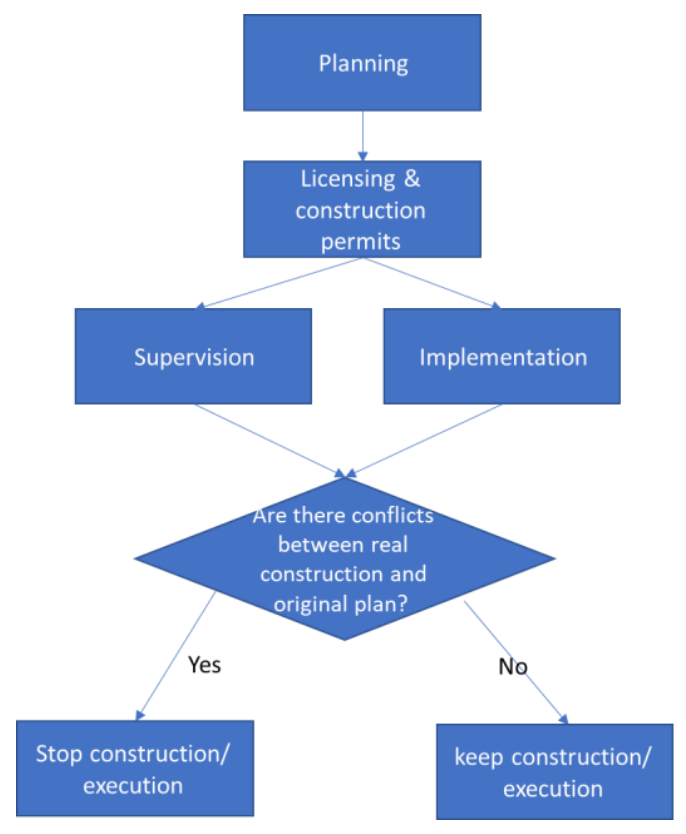

Figure 4. Conventional stages prior to construction involving the use of multi-purpose sustainable LMS.

An urban (or any) plan would not be executed/implemented without licensing. Supervision is performed all the way simultaneously to implementation. If any conflict between implementation and planning is detected through supervision, execution process might be obstructed, otherwise construction would be launched. If the procedure depicted in Figure 4 ends in construction, implementation or execution, it should be then followed by registration (as was illustrated in Figure 1).

For large scale projects, it is aspired that these procedures would be automatically done, which cannot happen without interactive, continuous real-time updating of databases, meaning that integrating the time dimension in LMS would be crucial.

An urban planning authority collaborates with at least three departments responsible for different tasks (Figure 5), namely: supervision, licensing and planning. For performing these tasks, the departments should collaborate and correspond during the entire time of a project. A multi-purpose MLMS system should fit for those agencies requirements, queries, databases, data structure and procedure. Such a system should be interactive, conscious/alert to modifications/alteration/ adjustments - and up to date.

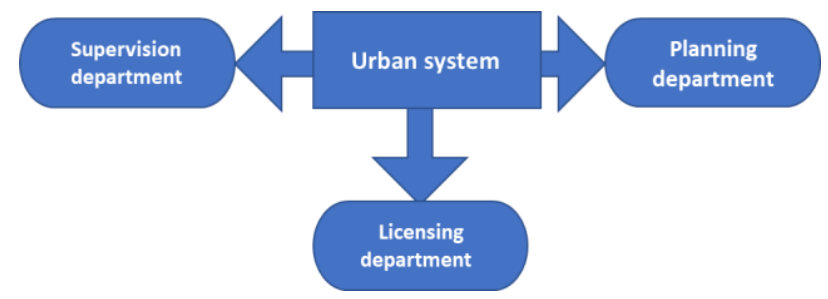

Figure 5. The three departments collaborating with an urban planning authority (system). 
3.7.4 Queries: new queries arise in MLMS. A potential 3D query is finding all the buildings that exist in a given radius and calculating their volume. An expected 4D query is calculating the rate of density growth over the last ten years. A possible 5D query is inserting a new volumetric spatial parcel in a MLMS, as illustrated in Figure 6.

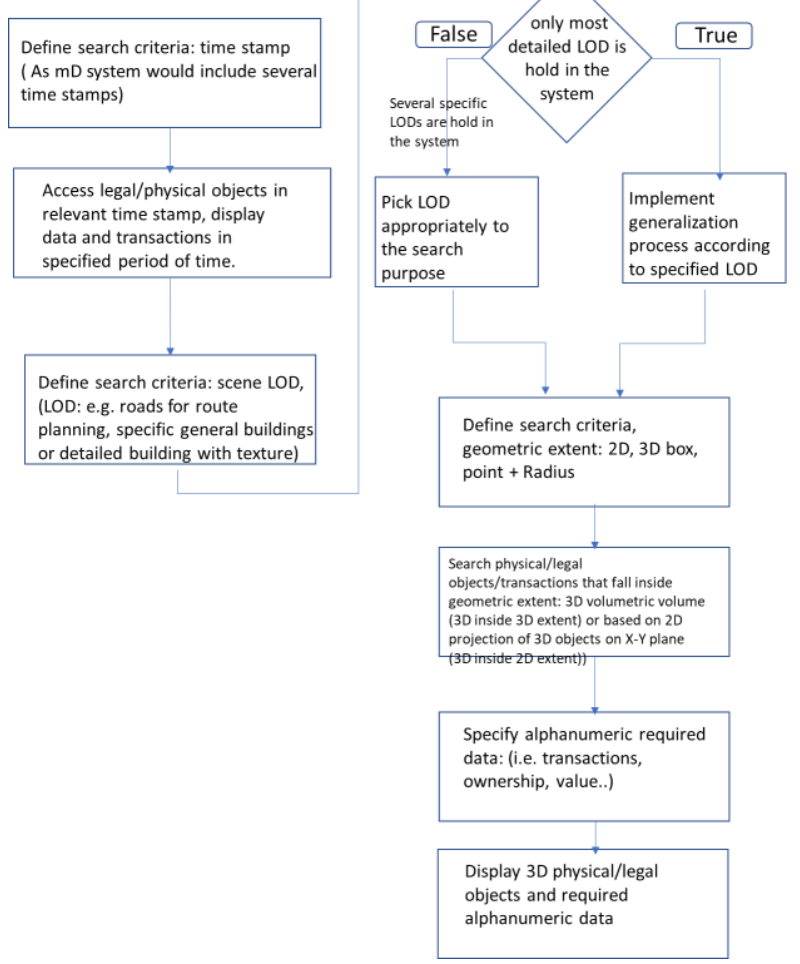

Figure 6. Illustrating a process of inserting a new volumetric spatial parcel in a MLMS.

\section{CONCLUSIONS}

This study is among the few studies implementing Delphi methodology in the field of land management. We follow Delphi approach since we aspire to set the requirements and expectations of MLMS based on potential stakeholders' perspective. Interviewing experts who have different experiences and come from diverse fields and countries reveals new ideas, highlighting issues that were not emphasized until now, some of which - if adopted - could change the whole research direction of LMS.

For instance, a respondent claimed that, based on his experience, MLMS cannot exist without multi-dimensional urban planning systems, implying that it would be wise to pause research on MLMS, and focus instead on developing multidimensional urban planning systems. Although there was a consensus on some matters, respondents' opinions varied, and they disagreed on specific issues. For example, they disagreed on the difficulty related to the managing of large volumes of data required for MLMS; some argue that managing big data would not be considered as a problem in a time when technological facilities are rapidly developing, while others claimed that despite the technical advancement, MLMS require collecting, cleaning, formatting and reorganizing massive volumes of data, which means investing time and resources just on the minor affairs, then only moving to analysis stage. Carrying out the tedious work of extracting massive volumes of data can be exhausting and challenging, even if relevant organizations are technologically accessed; for overcoming that, machine learning might be considered. Among the topics that experts agreed on are data sources. In their opinion, VGI and crowdsourcing lack maturity at this stage, and cannot be trusted to be a primary data source, but they may be used to complement other conventional sources, such as photogrammetry.

Overall, the preliminary analysis of the responses shows the importance and cruciality of creating multi-purpose MLMSs. Updates in data structure, classes and fields, as well as functionalities, queries and processes were suggested based on this analysis. Additional responses would be soon collected and analysed for expanding these preliminary results.

Large-scale projects are expected to be required for sustainably, serving future needs of land management in a time when population density reaches its peak and land resource become scarcer. This would stimulate the creation of multi-purpose MLMSs, in which data structure and classes should supply the requirements of various arenas; diversity of procedures and functionalities should be provided in MLMS, fitted for different end-users, written in programming languages that are easy to be converted to other specific languages used in relevant arenas. These mean that creating multi-purpose MLMS is a complex task, and more research and pilots should be performed in technical and conceptual topics concerned with MLMS. The option of unifying different agencies involved in land management processes should also be considered and investigated.

\section{REFERENCES}

Allard, J., 2009. The Design of Public Transport Maps-Graphic elements and design operations in the representation of urban navigation systems. Dipartimento INDACO. Milano, Politecnico di Milano. Doctor.

Güting, R. H., \& Schneider, M., 2005. Moving objects databases. Elsevier

Harrie, L., Sarjakoski, L. T., \& Lehto, L., 2002. A variablescale map for small-display cartography. International Archives of Photogrammetry Remote Sensing and Spatial Information Sciences, 34(4), 237-242

Jaljolie, R., van Oosterom, P., \& Dalyot, s., 2018. Spatial Data Structure and Functionalities for 3D Land Management System Implementation: Israel Case Study. ISPRS International Journal of Geo-Information 7(10)

Krigsholm, P., Riekkinen, K., \& Ståhle, P., 2018. The Changing Uses of Cadastral Information: A User-Driven Case Study. Land, 7(3), 83.

Langran, G., 1992. Time in Geographic Information Systems (London: Taylor \& Francis)

Nebiker, S., Lack, N., \& Deuber, M., 2014. Building change detection from historical aerial photographs using dense image matching and object-based image analysis. Remote Sensing, 6(9), 8310-8336.

Stoter, J., Visser, T., van Oosterom, P., Quak, W., \& Bakker, N., 2011. A semantic-rich multi-scale information model for 
topography. International Journal of Geographical Information Science, 25(5), 739-763.

Salem, R., Saleh, S. A., \& Abdul-Kader, H., 2018. Intelligent Replication for Distributed Active Real-Time Databases Systems. INTERNATIONAL ARAB JOURNAL OF INFORMATION TECHNOLOGY, 15(3), 505-513.

Van Oosterom, P., \& Stoter, J., 2012. Principles of 5D modeling, full integration of $3 \mathrm{D}$ space, time and scale. In Geospatial World Forum, 23-27 April 2012, Amsterdam. Geospatial World Forum. 